\title{
Champ de contrainte dans les argilites de Tournemire. Mesures in situ et interprétation
}

\section{A. REJEB}

Institut de Radioprotection

et de Sûreté Nucléaire BP 17

92262 Fontenay-aux-Roses Cedex amel.rejeb@irsn.fr

\section{TIJANI}

École nationale supérieure des mines de Paris 35, rue Saint-Honoré 77305 Fontainebleau tijani@cges.ensmp.fr
Cette étude présente deux campagnes de mesures de contraintes par la méthode de fracturation hydraulique en forages dans les argilites du Toarcien du site de Tournemire (Aveyron). On montre que cette méthode, basée sur la détermination des contraintes normales agissant sur un certain nombre de fractures

d'orientations connues, s'applique bien dans les milieux argileux malgré la finesse des fractures préexistantes. Cependant, cette étude démontre l'importance et en même temps la complexité de l'interprétation de ces mesures. En effet, l'interprétation de mesures identiques obtenues lors des deux campagnes d'essais, donne deux champs de contraintes géostatiques probables dans le site. Par ailleurs, les résultats des modélisations par éléments finis permettent d'une part de déterminer le champ de contrainte compatible avec les équations d'équilibre et les mesures in situ et d'autre part de quantifier l'influence de la topographique sur ce champ de contrainte dans le massif de Tournemire.

Mots clés : mesure de contraintes, fracturation hydraulique, forage, argilite, modélisation, éléments finis, effet topographique.

\section{Stress field in the Tournemire argillaceous. In situ measurements and interpretation}

This study presents two test series of stress measurements using hydraulic fracturing method in boreholes in the Toarcian shale at Tournemire site (Aveyron). It is shown that this method. based on the determination of the normal stresses supported by some fractures of known orientations, is well adapted at the argillaceous formations despite of the fineness of the preexisting fractures. Nevertheless, this study shows the importance and at the same time the complexity of the measurements interpretation. Indeed, the interpretation of the same measurements obtained during the two test series, gives two likely in situ stress fields. By another way, the finite element modelling results allow the determination of the stress field consistent with the balance equations and the in situ measurements. This modelling allows also the estimation of the topography effect on the stress field at the Tournemire rock mass.

Key words : stress measurements, hydraulic fracturing, borehole, shale, modelling, finite elements, topography effect. 


\section{Introduction}

La question de la gestion des déchets hautement radioactifs se pose à moyen et long terme étant donné que leur activité radiologique peut durer plusieurs centaines de millions d'années. Actuellement, une des solutions envisageables consiste à placer ces déchets à l'intérieur d'ouvrages souterrains édifiés à grande profondeur, dans un milieu limitant au mieux la dispersion des éléments radioactifs. Plusieurs roches sont donc étudiées parmi lesquelles les roches argileuses raides ou argilites.

La stabilité mécanique d'un tel ouvrage dépend des propriétés de la roche et de l'environnement géologique. Ce problème, classique dans le domaine du génie civil, devient beaucoup plus difficile dans le domaine du stockage de déchets nucléaires à cause de l'échelle de temps considérée.

Le problème mécanique posé par un stockage de déchets radioactifs ne se limite pas à la seule question de sa stabilité. Il s'agit d'évaluer la nature, l'étendue et l'évolution à long terme des perturbations créées par l'excavation. Les argilites possèdent des qualités mécaniques (rigidité et résistance importantes) et physicochimiques (faible perméabilité, capacité de rétention des radioéléments) a priori intéressantes pour le stockage, La construction d'un ouvrage souterrain risque d'altérer ces « bonnes » propriétés dans une certaine zone autour de l'ouvrage.

L'Institut de radioprotection et de sûreté nucléaire (IRSN) assure une mission de recherche et d'expertise auprès des pouvoirs publics, en particulier au sujet du stockage profond. Pour consolider ses connaissances sur les propriétés de confinement des matériaux argileux, il a sélectionné le site de Tournemire qui lui sert de station expérimentale. Un vaste projet de recherche, regroupant la géologie, I'hydrogéologie et la géochimie, y est mené depuis 1988 (Boisson et al., 1988). Un programme d'études géomécaniques a été développé en 1998 (Rejeb, 1999) et concerne les trois volets : essais au laboratoire, essais in situ et modélisations/validations.

Cet article présente les mesures de contraintes dans le site de Tournemire, leurs interprétations et la modélisation associée. Plus précisément, nous présentons tout d'abord les résultats de deux campagnes de mesures de contraintes par la méthode de fracturation hydraulique en forages qui confirment une fois de plus la complexité de ces mesures. Ensuite, les résultats de la modélisation par éléments finis permettent de quantifier l'influence de la topographique sur le champ de contrainte dans le massif de Tournemire.

\section{2}

\section{Description du site}

Le site de Tournemire a été choisi pour la facilitè d'accès que procure un ancien tunnel ferroviaire de $1885 \mathrm{~m}$ de long, traversant une couche argileuse de $250 \mathrm{~m}$ d'épaisseur. Ce site correspond à une structure monoclinale simple formée, à grande échelle, par trois grandes couches : une couche inférieure calcaire et dolomitique d'environ 300 m d'épaisseur (Hettangien, Sinémurien et Carixien), la couche argileuse constituée des formations du Toarcien et Domérien et une couche supérieure calcaire et dolomitique de plus de $300 \mathrm{~m}$ (Bajocien et Bathonien). La couche argileuse est encadrée par deux aquifères calcaires : l'aquifère de l'Aalénien dans la partie supérieure et l'aquifère régional du Carixien dans sa partie inférieure. L'ensemble de ces couches jurassiques est affecté vers le nord du site par une grande faille régionale, la faille du Cernon (Fig. 1).

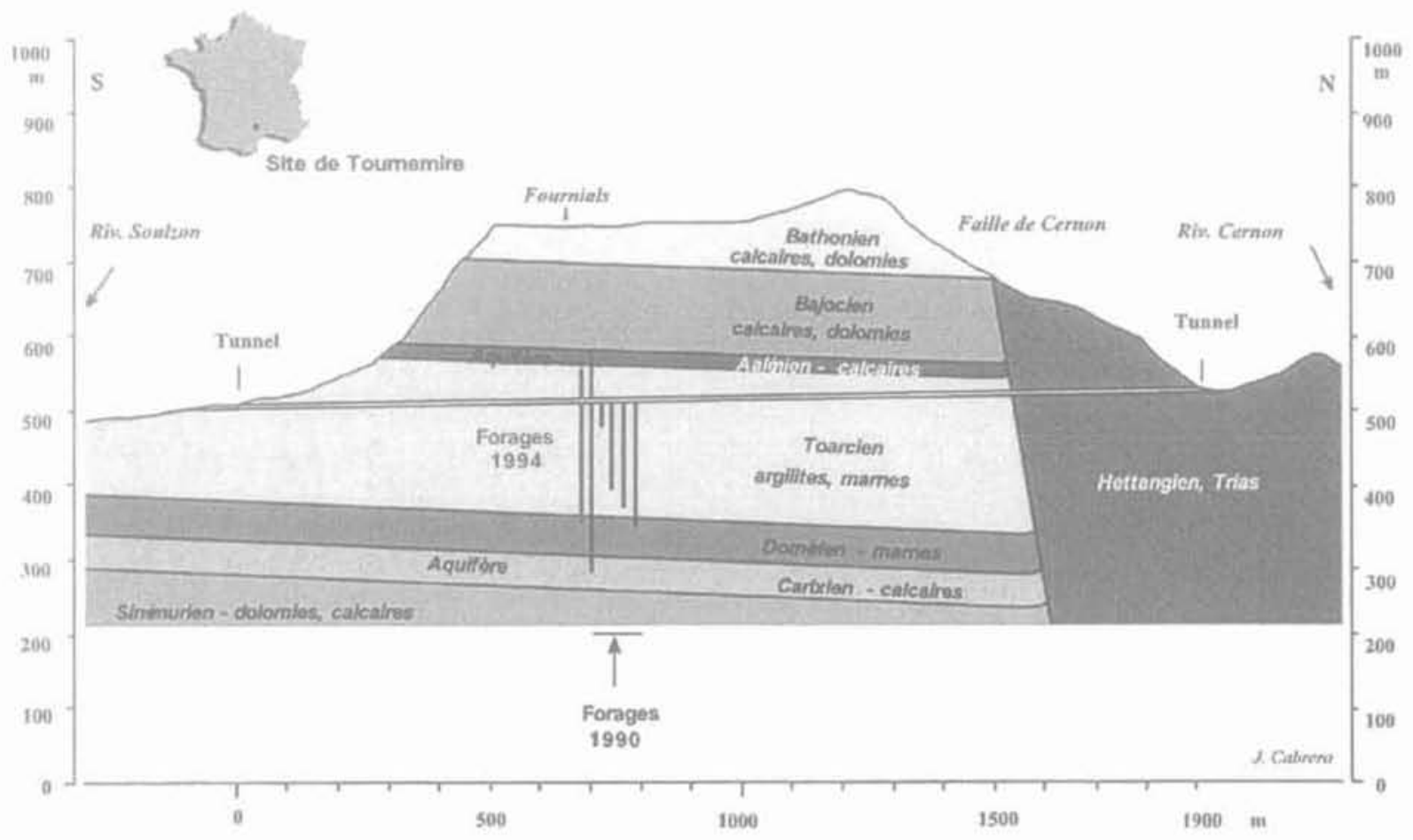

FIG.1 Coupe géologique du site de Tournemire.

Geological cross-section of the Tournemire site. 
Une campagne de huit forages rayonnants autour de l'axe du tunnel a été réalisée en 1994 (Fig. 2). Cette campagne a mis en évidence la présence d'une faille principale avec une fracturation associée d'origine tectonique affectant le milieu argileux ainsi que la présence d'eau dans quelques fissures. Suite à ces constatations, deux galeries de $30 \mathrm{~m}$ de long, dites Est et Ouest, ont été creusées en 1996 perpendiculairement à l'ancien tunnel (Fig. 2). L'implantation de ces deux galeries a été judicieusement choisie, afin que la galerie Ouest intercepte la faille principale et la galerie Est soit dans une zone saine.

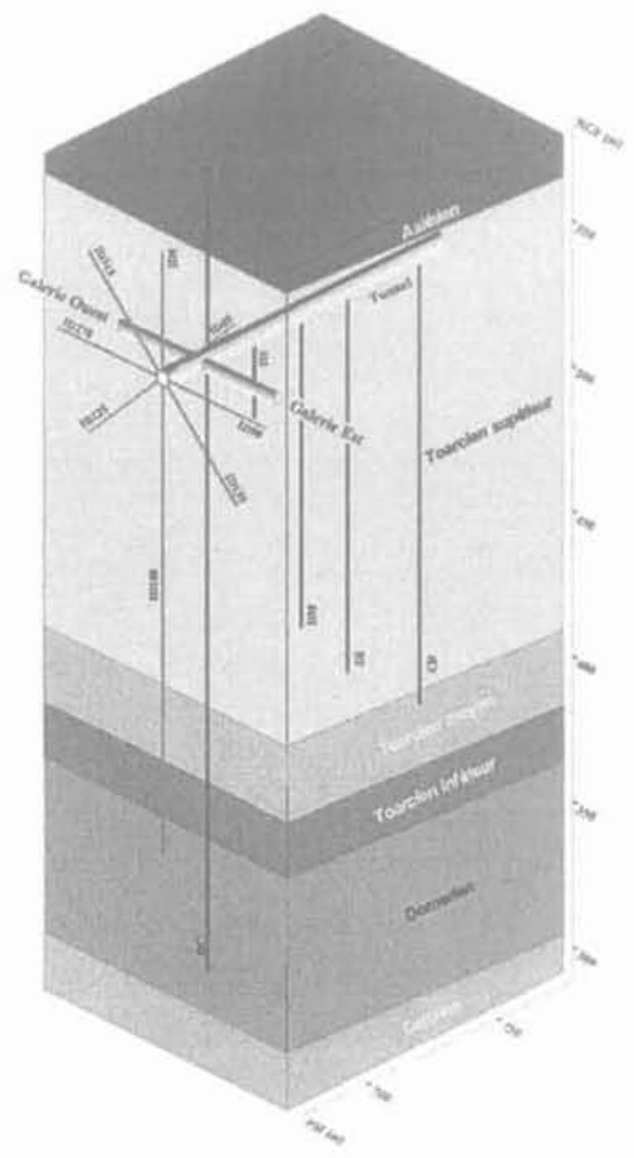

Fic. 2 Bloc diagramme de la zone des forages et des galeries du site de Tournemire.

Block-diagram of the boreholes and drifts area in the Tournemire site.

D'un point de vue géotechnique, le site de Tournemire présente deux importants éléments de comparaison entre:

- un tunnel creusé il y a cent ans et deux galeries récentes, notamment concernant l'évolution dans le temps des zones perturbées par le creusement:

- deux galeries creusées dans un contexte structural différent (zone saine et zone fracturée).

Dans le cadre du projet Tournemire, une synthèse détaillée des programmes de recherche et des résultats obtenus a été réalisée par Cabrera et al. (2001).

Les mesures de contraintes, objet de cette publication, ont été réalisées dans le forage vertical ID 180 et le forage incliné ID 225 au PM 675, au droit du lieu-dit « la ferme des Fournials ». La cote du tunnel au PM 675 est de 516 NGF alors que la cote de la surface du sol au droit du site est estimée à 751 NGF, soit 235 m au-dessus des têtes de forages (Fig. 1). Il est ainsi important de noter la topographie du site de Tournemire.

\section{3}

\section{Rappel des méthodes de mesures de contraintes par fracturation hydraulique}

Les premiers essais de fracturation hydraulique en forage ont été mis en ceuvre dans le but d'accroitre la productivité des puits pétroliers. Il s'agissait d'augmenter la perméabilité de l'encaissant par rupture de la roche en injectant des fluides dans une portion de forage isolé (Clarck, 1949). Fairhurst (1964) fut parmi les premiers à préconiser l'utilisation de la fracturation hydraulique comme méthode de détermination du tenseur des contraintes locales. Cette méthode est actuellement la plus courante pour estimer l'état des contraintes en grande profondeur, particulièrement dans les milieux cristallins.

On distingue la méthode de fracturation hydraulique classique (Haimson et Fairhurst, 1969) et la méthode HTPF (Hydraulic Tests on Pre-existing Fractures) développée par Cornet et Valette (1984). La méthode HTPF est une généralisation dé la méthode classique. Ces méthodes ont étẻ déjả décrites dans plusieurs références et notamment dans les thèses de Burlet (1991) et de Willeveau (1997) et ne sont que brièvement rappelées ici.

\section{1}

\section{Méthode de fracturation hydraulique classique}

Cette méthode consiste à isoler une portion du forage entre obturateurs étanches à la profondeur désirée et à la mettre sous pression hydrostatique croissante jusqu'à ce qu'apparaisse, pour une valeur particuliêre $\mathrm{P}$. de la pression, une fracture. Celle-ci est ensuite propagée sur une certaine distance par injection de fluide puis, à l'arrêt du pompage, le forage est maintenu sous pression. La pression chute alors brutalement, puis plus lentement et enfin tend à se stabiliser (Fig. 3), La pression de fermeture $\mathrm{P}$, correspond à la transition entre la chute rapide et lente de la pression et caractérise la fermeture mécanique de la fracture. L'amplitude de la contrainte principale mineure est ainsi déterminée directement par $\sigma_{n}=P_{r}$ Les courbes pression-temps et la détermination de l'orientation de la fracture au niveau du forage (Fig. 3) permettent de déterminer les composantes horizontales du tenseur contrainte suivant la solution du cylindre creux et infini. percé dans un milieu linéairement élastique et isotrope (Hiramatsu et Oka, 1962). La contrainte $\sigma_{4}$ supposée verticale est définie par le poids des terres.

Les essais de fracturation hydraulique permettent une bonne détermination de la direction de la contrainte horizontale majeure et de l'amplitude de la contrainte principale mineure. Néanmoins, ils ne fournissent pas correctement l'amplitude de la contrainte principale horizontale majeure du fait des problèmes liés à la pression interstitielle (Burlet, 1991). La méthode HTPF permet de pallier ces limites. 


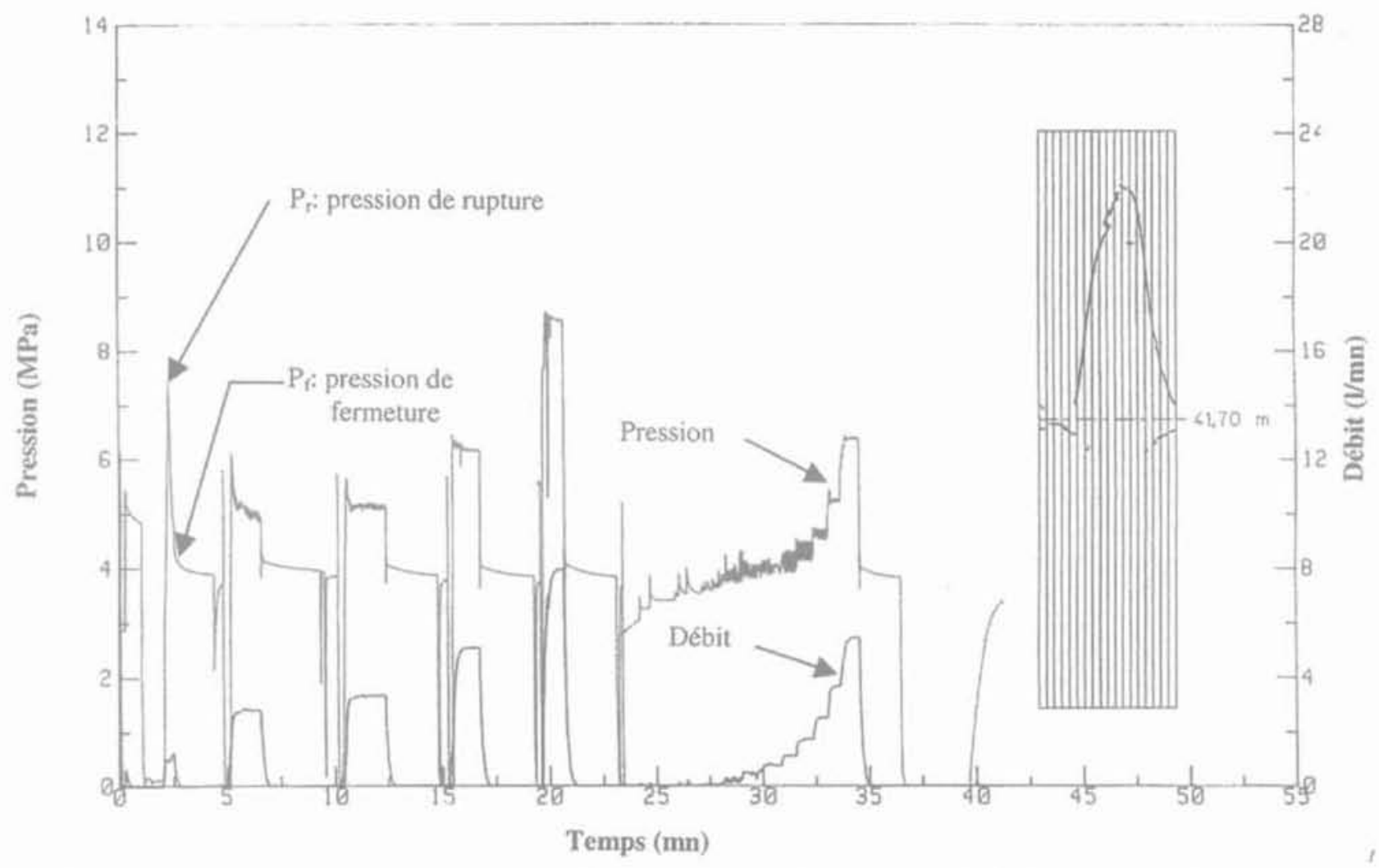

FG.3 Exemple d'une courbe pression-débit-temps avec l'empreinte de la fracture testée au cours de la campagne d'essais de 1995 à Tournemire.

Example of pressure-flow rate-time curve and the trace fracture tested during the 1995 test series at Tournemire site.

\section{2}

\section{Méthode HTPF}

La méthode HTPF correspond à une généralisation de la méthode de fracturation hydraulique classique. Elle permet d'échantillonner toutes les directions du champ de contrainte en mesurant la contrainte normale supportée par des plans de fractures naturelles d'orientations quelconques et différentes. La difficulté réside dans la sélection des intervalles de test hydraulique où une fracture unique existe. L'orientation des fractures testées peut être déterminée à la paroi du forage au moyen d'un obturateur d'impression. Cependant, ce procédé de prise d'empreinte nécessite de nombreux va-et-vient d'outils dans le forage ce qui rallonge la durée de la campagne et augmente l'incertitude sur le positionnement de l'empreinte de la fracture testée. Les outils de diagraphies géophysiques et les divers procédés d'imagerie sont mieux adaptés à ce type de reconnaissance (Wileveau, 1997).

Les essais supposent qu'une fracture unique a été ouverte. Dans le cas où plusieurs fractures se seraient ouvertes, il importe de déterminer la chronologie d'ouverture des fractures, car dans ce cas, seul le premier essai d'ouverture permet de remonter au champ des contraintes naturelles. A partir des mesures de contrainte normale et d'orientation des fractures, des méthodes d'inversion (moindres carrés ou algorithmes de Monte-Carlo) permettent de calculer les composantes du tenseur des contraintes locales.

Le matériel nécessaire pour ces essais hydrauliques ainsi que leur mise en œuvre sont assez complexes. Par conséquent, la précision du champ de contrainte cal- culé dépend de l'outil de mesure utilisé, du nombre d'essais effectués, de l'environnement naturel et de la méthode de résolution et d'interprétation.

\section{4}

\section{Première campagne d'essais (1995)}

\section{1}

\section{Description}

Cette première campagne d'essais a étẻ réalisée en juillet 1995 par la société ANTEA (Antoinet et Bertrand, 1996). Il s'agit de onze essais dans le forage vertical ID 180 entre les profondeurs $41 \mathrm{~m}$ et $143 \mathrm{~m}$ comptées à partir de la tête du forage. Quatre autres essais ont été réalisés entre les profondeurs $26 \mathrm{~m}$ et $31 \mathrm{~m}$ dans le forage incliné ID 225. Deux essais dans ce dernier forage n'ont pas permis de réaliser des mesures du fait du contournement des obturateurs. Sur l'ensemble de la campagne d'essais, uniquement trois essais de fracturation hydraulique classique ont été réalisés. Le reste des essais HTPF a été réalisé au niveau d'une fracture naturelle unique et inclinée à l'exception de trois essais dont les empreintes présentent les traces de plusieurs fractures inclinées différemment.

Un exemple de courbe pression et débit d'injection en fonction du temps avec le relevé effectué au packer à empreintes de la fracture testée est présenté dans la figure 3. L'ensemble des courbes, présentant des changements de courbure bien marqués en raison de la faible perméabilité des argilites de Tournemire, a per- 
mis de déterminer les pressions de fermeture sans ambiguïté. On note aussi une bonne reproductibilité des mesures de pression au cours d'un cycle de mesures.

\section{2}

\section{Interprétation}

Le tenseur des contraintes locales qui s'exerce sur une fracture i, définie par l'orientation de sa normale n, et qui supporte la contrainte normale $\sigma_{\mathrm{ni}}$ s'écrit dans lé repère géographique local :

$$
[\sigma(\mathrm{x})] \cdot n_{1} \cdot n_{\mathrm{i}}=\sigma_{\mathrm{n}}
$$

On suppose que le tenseur [ $\sigma(x)]$ est uniforme dans tout le volume affecté par la fracture et que la pression de fermeture $\mathrm{P}$, est une mesure de la contrainte $\sigma$. Le problème est donc de déterminer les six composantes de $[\sigma(x)]$ en tous points $x$ du volume rocheux qui

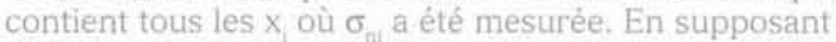
que les variations verticales de contraintes sont prépondérantes aux variations latérales, l'équation (1) devient:

$$
\left[\sigma\left(z_{i}\right)\right] \cdot n_{i} \cdot n_{i}=\sigma_{n i}
$$

où $[\sigma(z)]$ est la matrice de contraintes à la profondeur $z$ de la fracture $\mathrm{i}$.

La résolution d'un tel système dans le cas le plus général nécessite de nombreuses mesures de $\sigma_{n:}$.

Afin de simplifier le système et pouvoir le résoudre avec les mesures disponibles tout en utilisant une méthode d'inversion généralisée, les hypothèses suivantes ont été adoptées:

- dépendance linéaire de $[\sigma(z)]$ avec la profondeur ; - l'une des directions principales est voisine de la verticale ;

- les variations de contraintes dans l'intervalle de mesures sont faibles.

\section{3}

\section{Résultats}

Les variations des contraintes principales avec la profondeur, calculées à partir de la solution de l'inversion des données de mesures, sont présentées dans la figure 4. Les lignes en trait discontinu donnent les incertitudes sur les valeurs des contraintes horizontales $\sigma_{H}$ et $\sigma_{\mathrm{h}}$ calculées. La contrainte principale majeure est la contrainte verticale $\sigma_{y}$; elle est inférieure au poids des terres estimé à partir de la densité des argilites de Tournemire $\left(25,5 \mathrm{kN} / \mathrm{m}^{3}\right)$. La contrainte principale intermédiaire $\sigma_{H}$ augmente légèrement alors que la contrainte principale mineure $\sigma_{\mathrm{h}}$ diminue avec la profondeur. Les diverses campagnes d'essais, présentées dans Burlet (1991), montrent que $\sigma_{h}$ et $\sigma_{\mu}$ augmentent généralement avec la profondeur dans les formations cristallines et diminuent dans le cas d'un site sédimentaire.

On retient de cette campagne d'essais que l'état de contrainte dans le massif est quasi isotrope dans la partie supérieure de la zone testée avec $\sigma_{\mathrm{v}}=\sigma_{H}=\sigma_{\mathrm{h}}=4,32$ $\pm 1,13 \mathrm{MPa}$ et une direction de $\mathrm{N} 72^{\circ}$ pour la contrainte $\sigma_{\mathrm{H}}$. Cette orientation se raccorde mal avec ce qu'on sait sur l'orientation des contraintes tectoniques à l'échelle régionale $\left(\mathrm{N} 160^{\circ}\right)$ dans Cabrera et al. (2001).

On explique la différence entre la contrainte verticale et le poids des terres par la présence du tunnel, qui

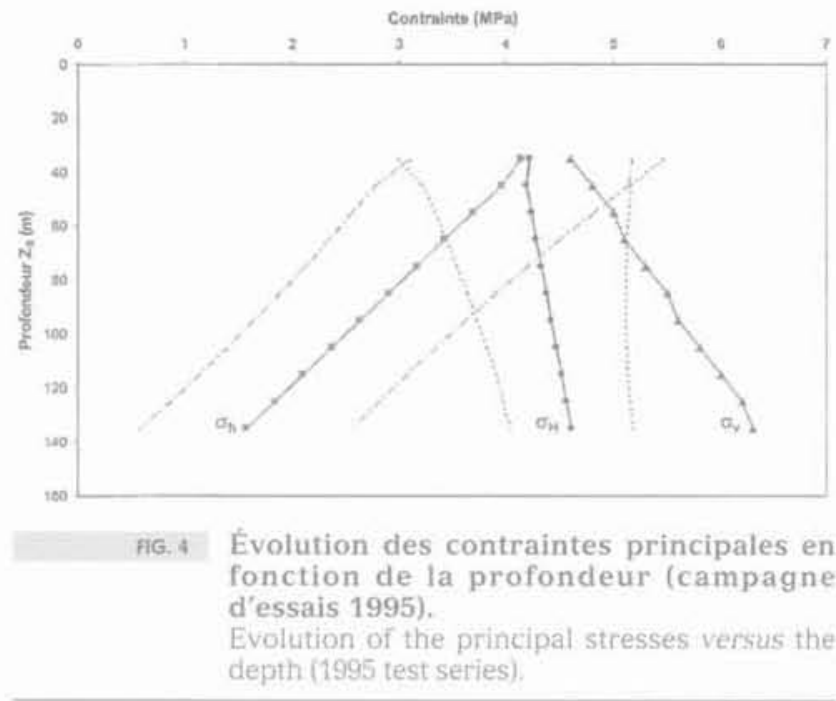

peut modifier non seulement les intensités des contraintes mais aussi leurs directions principales, et/ou l'effet de la topographie du massif. Seul un plus grand nombre de mesures pourrait permettre de mieux préciser ces résultats préliminaires.

\section{Deuxième campagne d'essais (1999)}

\section{1}

\section{Description}

Pour vérifier et compléter les résultats de la première campagne d'essais, I'Institut de Physique du Globe de Paris (IPGP), en association avec la société GEOSTRESS, a réalisé une deuxième campagne d'essais en 1999. Il s'agit de huit essais réalisés dans le forage ID 180 entre les profondeurs $24 \mathrm{~m}$ et $135 \mathrm{~m}$ et quatre essais dans le forage ID 225 entre les profondeurs $25 \mathrm{~m}$ et $34 \mathrm{~m}$. L'ensemble de la campagne concerne des essais HTPF avec un seul essai par fracturation hydraulique classique (Wileveau, 1999; Cornet, 2000). Les quatre essais les mieux réussis en 1995 ont été repris lors de cette campagne, afin de s'assurer de la répétitivité des dites mesures.

Afin d'éviter que des essais donnent lieu à de multiples fractures, on a prévu d'utiliser une sonde HTPF munie d'un système d'imagerie électrique qui permet théoriquement d'identifier en temps réel les fractures ouvertes par l'injection d'eau. Malheureusement, ce procédé n'a pas fonctionné étant donné le faible contraste de résistivité entre l'image électrique avant et après l'injection hydraulique. Ce qui a engendré la réalisation de cette campagne d'essais en deux temps : en juin 1999 ont été réalisés les essais HTPF et en septembre 1999 ont été déterminées les orientations des fractures testées auparavant à l'aide d'un packer d'empreinte.

\section{2}

\section{Interprétation}

L'objectif est d'obtenir une détermination explicite complète de toutes les composantes du tenseur de contrainte autour du tunnel, sans hypothèses a priori 
sur les directions et les poids des terres. En effet, si l'on assimile les couches calcaires à une plaque rigide chargeant les argilites et les marnes du Toarcien, il apparait, selon les principes classiques de la mécanique des sols, que la distribution de contrainte verticale à la base de l'Aalénien ne peut être évaluée simplement (Craig. 1987). De plus, pour ce qui concerne les variations spatiales du champ de contrainte, il a été montré qu'en milieu sédimentaire ces variations peuvent ne pas être linéaires avec la profondeur (Evans et al., 1989 ; Cornet et Burlet, 1992). Dans le cas du site de Tournemire, cette non-linéarité ne permet pas d'interpréter les mesures selon la méthode HTPF avec une inversion globale des données.

Pour circonvenir l'ensemble de ces difficultés, I'interprétation des mesures de cette deuxième campagne a été menée pas à pas en combinant la méthode de fracturation hydraulique classique et la méthode HTPF tout en considérant les données les plus cohérentes et les moins ambiguës.

\section{3}

\section{Résultats}

La figure 5 donne la variation des trois composantes principales des contraintes avec la profondeur dans l'intervalle testé. On ne peut pas mettre en évidence une augmentation ou une diminution systématique des contraintes principales horizontales avec la profondeur ; les variations latérales dominent ces résultats. Ces variations peuvent ètre attribuées à la présence de fractures préexistantes remplies de calcite entre les profondeurs $30 \mathrm{~m}$ et $50 \mathrm{~m}$. Le gradient vertical de la composante verticale représentée sur la figure 5 est d'environ $0,026 \mathrm{MPa} / \mathrm{m}$; il convient d'éviter d'extrapoler cette valeur au-delà des profondeurs testées du fait du changement de nature du matériau dans le massif et de la topographie.

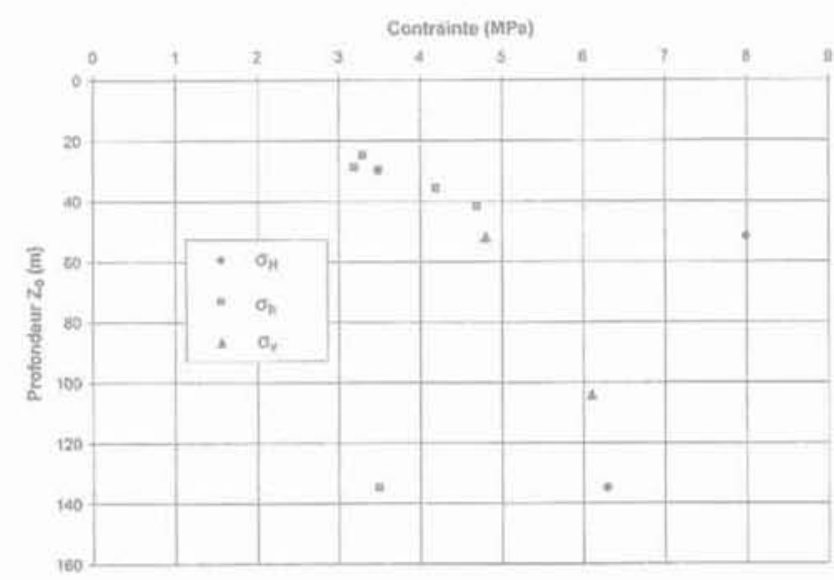

FG. 5 Évolution des contraintes principales en fonction de la profondeur (campagne d'essais 1999).

Evolution of the principal stresses versus the depth (1999 test series).

Au voisinage du tunnel, l'amplitude et l'orientation de chacune des trois composantes du champ des contraintes locales sont :

$\sigma_{\mathrm{h}}=2,1 \pm 1,0 \mathrm{MPa}$, orientée $\mathrm{N} 72^{\circ} \pm 15^{\circ}$ E et fait un angle de $10^{\circ}$ avec l'horizontale; $\sigma_{v}=3,8 \pm 0,4 \mathrm{MPa}$, orientée $\mathrm{N} 72^{\circ} \mathrm{E}$ et fait un angle de $10^{\circ}$ avec la verticale :

$\sigma_{\mathrm{H}}=4,0 \pm 2,0 \mathrm{MPa}$, orientẻe $\mathrm{N} 162^{\circ} \pm 15^{\circ} \mathrm{E}$.

$L^{\prime}$ amplitude de la composante verticale inférieure aux poids des terres ( $6 \mathrm{MPa}$ ) ainsi que son inclinaison reflètent probablement les effets de la topographie du site de Tournemire.

La direction de la contrainte horizontale majeure observée, est significative des contraintes tectoniques régionales, du fait de l'absence de structure significative au voisinage du site. Cependant, l'absence d'essais HTPF sur des fractures préexistantes d'orientations suffisamment différentes n'a pas permis de déterminer de façon précise l'amplitude de la contrainte horizontale majeure.

\section{6}

\section{Comparaison des deux campagnes d'essais}

Les deux campagnes d'essais pour les mesures des contraintes dans le site de Tournemire s'accordent bien en ce qui concerne les amplitudes des contraintes normales s'exerçant sur les fractures testées et diffèrent notablement en ce qui concerne les orientations de ces fractures et le champ de contrainte autour du tunnel.

\section{1}

\section{Contraintes normales locales}

Le tableau I regroupe les résultats des mesures des contraintes normales $\left(\sigma_{n}\right)$ obtenues lors des quatre essais réalisés pendant les deux campagnes de 1995 et 1999. La cote $Z_{0}$ représente la profondeur par rapport à la tête du forage. Ces résultats nous permettent de constater que la technique des essais hydrauliques pour déterminer l'amplitude de la contrainte normale supportée par les fractures dans les argilites s'est révélée tout à fait fiable. En effet, avec du matériel et des équipes différentes, à des périodes différentes, on retrouve des amplitudes identiques avec une légère variabilité de 0,2 à $0,3 \mathrm{MPa}$. Cependant, des résultats différents ont été obtenus pour les orientations des quatre fractures testées durant les deux campagnes d'essais; ce qui peut influer considérablement la détermination du champ de contrainte.

TABLEAUI Comparaison des contraintes normales mesurées en 1995 et en 1999 dans le forage ID 180.

Comparison between normal stresses measured during 1995 and 1999 in ID 180 borehole.

\begin{tabular}{c|c|c|c|c} 
& \multicolumn{2}{|c|}{ Mestures 1995 } & \multicolumn{2}{c}{ Mesures 1999 } \\
\hline$N^{\circ}$ essai & $Z_{0}(\mathrm{~m})$ & $\sigma_{0}(\mathrm{MPa})$ & $\mathrm{Z}_{0}(\mathrm{~m})$ & $\sigma_{0}(\mathrm{MPa})$ \\
\hline 1 & 41,70 & $4,5 \pm 0,2$ & 41,70 & $4,75 \pm 0,21$ \\
\hline 2 & 52,25 & $4,4 \pm 0,2$ & 52,30 & $3,81 \pm 0,21$ \\
\hline 3 & 104,30 & $5,8 \pm 0,2$ & 104,60 & $6,09 \pm 0,21$ \\
\hline 4 & 134,95 & $3,3 \pm 0,2$ & 135,30 & $3,47 \pm 0,13$ \\
\hline
\end{tabular}


On constate aussi que le creusement des deux galeries perpendiculairement à l'ancien tunnel en 1996 à une quinzaine de mètres des forages où ont été réalisés les essais hydrauliques, n'a pratiquement pas induit de perturbations mécaniques significatives entre les profondeurs $40 \mathrm{~m}$ et $130 \mathrm{~m}$ en dessous du tunnel. En effet, les deux campagnes 1995 et 1999 réalisées respectivement avant et après le creusement des galeries, donnent les mêmes mesures.

\section{2}

\section{Champ de contrainte au niveau du tunnel}

Comme le montrent les résuitats précédents ( $\$ 4.3$ et 5.3) les deux campagnes d'essais ont conduit, à partir des mesures des contraintes normales et de l'orientation des fractures testées, à deux champs de contraintes différents. L'écart entre ces deux champs de contraintes est surtout important en ce qui concerne les directions des contraintes principales ; soit $90^{\circ}$ pour la contrainte horizontale intermédiaire. Ce qui démontre, d'une part, l'importance des résultats des orientations des fractures testées et, d'autre part, l'importante et la difficulté de l'interprétation des données obtenues par essais hydrauliques. Par ailleurs, on retrouve dans les deux campagnes l'effet topographique du massif sur l'amplitude de la contrainte verticale,

\section{7}

\section{Modélisation de l'effet de la topographie sur le champ de contrainte}

\section{1}

\section{Intérêt de la modélisation}

Afin de vérifier l'influence de la topographie du site sur les contraintes mesurées à Tournemire, une modélisation par élérnents finis a été réalisée. Cette modélisation permet d'estimer un champ de contrainte probable, compatible avec les équations différentielles d'équilibre, et qui sera comparé aux champs de contraintes proposés par les deux précédentes campagnes d'essais.

Le champ de contrainte dépend de la rhéologie, de la genèse, de l'hétérogénéité, de l'anisotropie, de la topographie du site... La modélisation du site de Tournemire a permis d'étudier l'influence de chacun de ces paramètres. Dans cet article, n'est présenté que l'effet de la topographie sur le champ de contrainte à Tournemire.

\section{2}

\section{Synthèse bibliographique}

Amadei et Stephansson (1997) ont présenté une revue bibliographique, bien fournie, sur les travaux de modélisations numériques et analytiques de l'effet de la topographie sur le champ de contraintes. Les principales caractéristiques de ces modélisations sont résumées ici :
- Absence totale de lien entre I'historique modélisé et les schémas que les géologues imaginent pour la genèse du massif rocheux. Cette faiblesse des modélisations est pratiquement inévitable dans la mesure où la genèse proposée par le géologue n'est informée que qualitativement.

- Usage fréquent de l'élasticité linéaire. Cette hypothèse sur le comportement des matériaux permet en effet de choisir un état de référence non contraint et d'appliquer sur le volume $V$ les forces de gravité en admettant que la frontière latérale de $V$ est soumise soit à un déplacement nul soit à des pressions tectoniques connues. Cette démarche suppose en plus que les paramètres élastiques ne changent pas durant la genèse et que le type de conditions aux limites est resté le même durant l'évolution du massif. Les déplacements entre l'état relâché et l'état final sont supposés suffisamment petits pour confondre la géométrie initiale (inconnue) et la géométrie finale y compris lorsque la surface libre n'est pas horizontale.

- Rareté de la comparaison entre les résultats des calculs et les mesures in situ. Les quelques études où l'on a procédé à une telle comparaison conduisent d'abord à une nette divergence entre théorie et réalité qui est ensuite réduite en modifiant les hypothèses des calculs.

\section{3}

\section{Description et données du modèle}

Il s'agit de deux modèles numériques bidimensionnels (déformations planes) en élasticité linéaire avec l'hypothèse des transformations infinitésimales. Les deux modèles diffèrent uniquement par l'état de référence et les densités des divers matériaux.

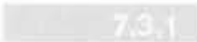 \\ Données géométriques}

La figure 6 présente la coupe verticale considérée et montre les divers faciès ainsi que l'emplacement des points de mesures où on a déterminé les contraintes, Cette coupe s'étend horizontalement sur $4 \mathrm{~km}$ et verticalement sur $2 \mathrm{~km}$; dimensions suffisantes pour représenter l'infini. En revanche, on n'a pas modélisé le tunnel qui est suffisamment loin des points de mesure

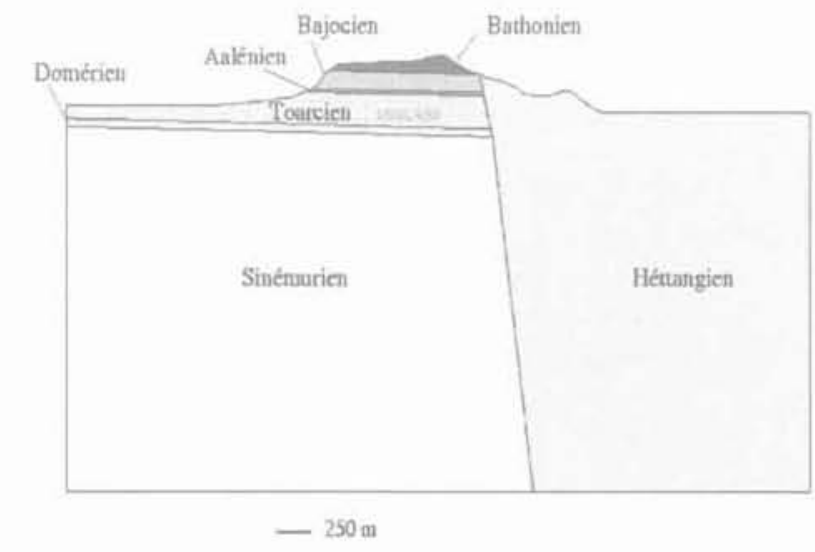

FG.6 Données géométriques des modélisations numériques.

Geometrical data for the numerical simulations. 
pour négliger l'effet de sa présence sur les contraintes. Plus précisément, le rapport entre le rayon du tunnel et la distance séparant le tunnel du point de mesure le plus proche est un nombre très petit vis-à-vis de 1 pour être autorisé à négliger l'effet de structure. D'ailleurs, cette hypothèse est largement confirmée par les mesures mentionnées dans le paragraphe 6.1.

\subsection{6}

\section{Létat de référence}

Dans le premier modèle, l'état de référence est relâché (approche classique). Le second modèle correspond à un schéma par lequel on imagine un état de référence sans relief avec des contraintes initiales connues puis une création du relief par érosion. L'état de contraintes de référence pour ce deuxième modèle est caractérisé uniquement par trois composantes : une contrainte verticale $\sigma_{\mathrm{y}}$ et deux contraintes horizontales $\sigma_{h}$ et $\sigma_{H}$. Ces trois composantes, variant linéairement en fonction de la profondeur, ont été déterminées en ajustant les contraintes obtenues à l'état final par le modèle sur les mesures des deux campagnes d'essais 1995 et 1999.

\section{7an:3}

\section{Les matériaux}

Le massif rocheux de Tournemire est constitué de 7 faciès. Mis à part, l'argilite du Toarcien, les six autres matériaux sont élastiques et isotropes. Dans le premier modèle, on a utilisé les masses volumiques et les paramètres élastiques, déterminés par des mesures et essais de laboratoire, Pour ces calcaires, la masse volumique moyenne est de l'ordre de $2600 \mathrm{~kg} / \mathrm{m}^{3}$ et le module d'Young est de l'ordre de $7800 \mathrm{MPa}$.

Par ailleurs, les essais triaxiaux en post-rupture réalisés sur des échantillons d'argilite du Toarcien montrent que le comportement du matériau est élastoplastique poursuivie par une rupture fragile. Le comportement élastique est analysé sur la base d'un matériau isotrope transverse décrit par la loi de Hooke généralisée (Lekhnitskii, 1963). Les cinq paramètres indépendants, utilisés dans les deux modèles, ont pour valeurs (Rejeb et al., 1999) :

$$
\begin{aligned}
& E_{1}=27680 \pm 4040 \mathrm{MPa} \text { et } v_{12}=0,17 \pm 0,03 \\
& E_{2}=9270 \pm 490 \mathrm{MPa} \text { et } v_{23}=0,20 \pm 0,03 \\
& G_{12}=3940 \pm 480 \mathrm{MPa}
\end{aligned}
$$

où $E_{\text {, }}$ et $E_{2}$ sont respectivement les modules d'Young dans les directions parallèle et perpendiculaire à la stratification. $G_{12}$ est le module de cisaillement dans le plan constitué par les directions parallèle et perpendiculaire à la stratification. Le coefficient de Poisson v caractérise la déformation dans la direction $\mathrm{j}$ due à une sollicitation dans la direction $\mathrm{i}$.

Dans le deuxième modèle, on a considéré une masse volumique identique pour les 7 faciès, égale à $1900 \mathrm{~kg} / \mathrm{m}^{3}$.

\section{3is}

\section{Les conditions aux limites}

Sur la frontière libre, représentant le relief, les forces extérieures sont nulles. Sur le bord horizontal inférieur, l'effort horizontal et le déplacement vertical sont nuls. Sur les deux bords verticaux, l'effort vertical et le déplacement horizontal sont nuls. La continuité des déplacements est imposée entre les différentes couches.

\section{4}

\section{Principaux résultats}

Il est intéressant de représenter graphiquement l'effet de la topographie par la densité ou le poids volumique apparent. Le poids volumique apparent est, au signe prés, la dérivée de la contrainte verticale par rapport à z. La différence entre les poids volumiques réel et apparent est la dérivée du cisaillement par rapport à la coordonnée horizontale x.

Avec les deux modèles, on remarque les mêmes phénomènes déjà observés par d'autres auteurs (Sturgul et al., 1976). Sous une montagne, on a une densité apparente plus faible que la densité réelle et ce parce que la montagne s'appuie sur le reste du massif rocheux au niveau de ses extrémités ; une sorte d'effet de voûte.

Les figures 7 à 9 illustrent les résultats du premier modèle. On trouve respectivement les zones d'intensité de la contrainte verticale (Fig. 7) et de l'écart entre densités réelle et apparente (Fig. 8) ainsi que le profil le
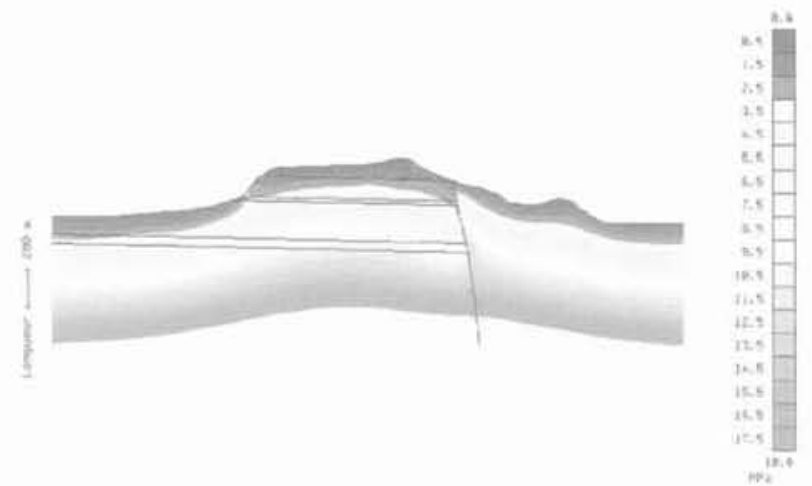

FlG.7 Répartition de la contrainte verticale (modèle 1). Vertical stress distribution (model 1).

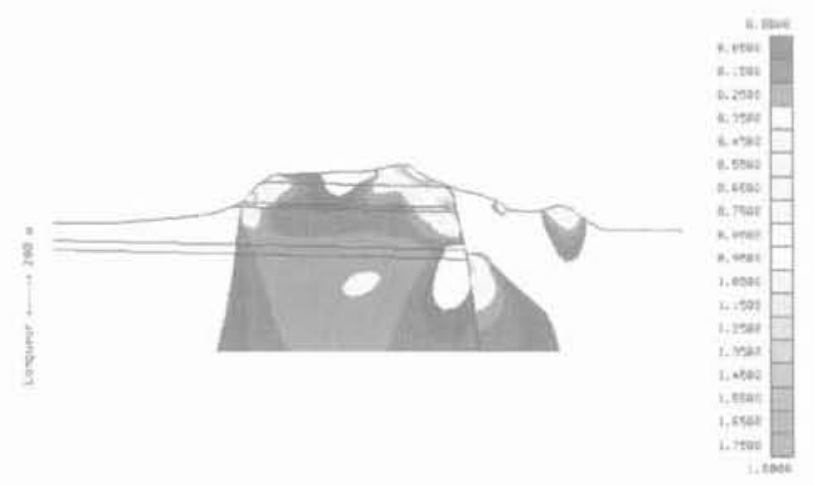

FIG.8 Répartition de la perte de densité (modèle 1). The decrease of the density distibution (model 1). 
long du forage ID180 des densités réelle, apparente et de leur différence (Fig. 9). Cette dernière figure montre clairement qu'au voisinage des points de mesure (entre les profondeurs $25 \mathrm{~m}$ et $140 \mathrm{~m}$ ) l'effet de la topographie est relativement faible (un maximum de $10 \%$ ).

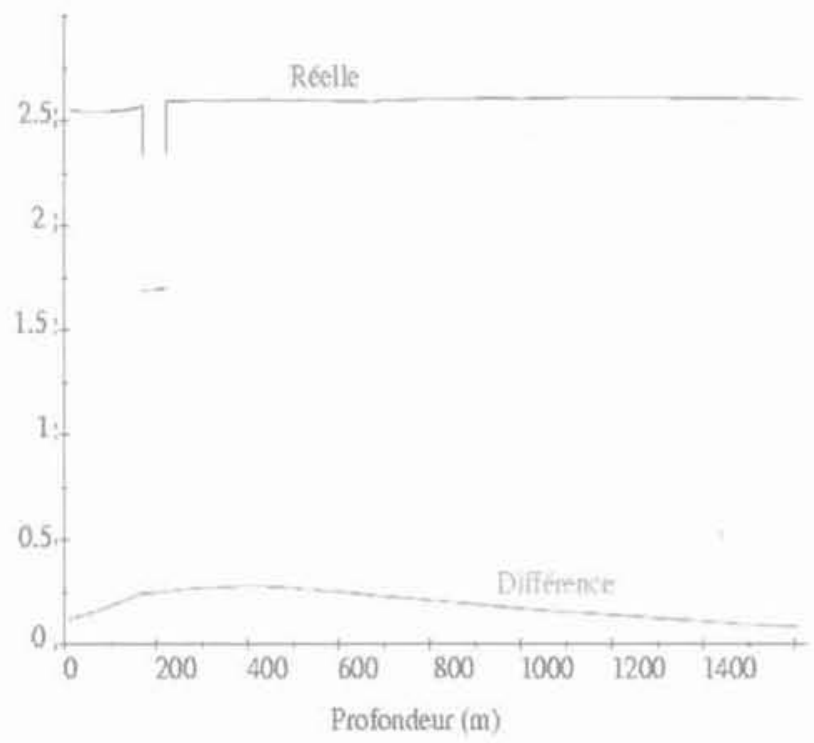

NG. 9 Densités réelle et apparente en fonction de la profondeur (modèle 1).

Actual and apparent densities versus the depth (model 1).

\section{8}

\section{Comparaison des calculs aux mesures dans le site}

\section{1}

\section{Contraintes normales locales}

L'analyse des résultats des contraintes le long du forage ID 180 obtenus par le premier modèle montre que la contrainte verticale calculée peut aller jusqu'à $8 \mathrm{MPa}$ à la profondeur $104 \mathrm{~m}$. Cette valeur est bien trop élevée par rapport aux mesures des contraintes normales dans le site (voir tableau 1 , essai $n^{\circ} 3$ ). Cela nous conduit alors à réduire les densités avec un rapport de l'ordre de 0,75 (modèle 1, réduit).

TABLEAUII Comparaison des contraintes normales mesurées et calculées en MPa.

Comparison between measured and computed normal stresses in $\mathrm{MPa}$.

\begin{tabular}{c|c|c|c}
\hline Mesures & Modèle 1 & $\begin{array}{c}\text { Modèle 1 } \\
\text { (réduit) }\end{array}$ & Modele 2 \\
\hline M95-3,3 & 6,6 & 5,0 & 3,5 \\
\hline M95-5,8 & 8,3 & 6,2 & 5,9 \\
\hline M95-4,4 & 5,8 & 4,4 & 3,4 \\
\hline M 95-4,5 & 4,4 & 3,3 & 4,5 \\
\hline M 99-3,5 & 6,6 & 5,0 & 3,9 \\
\hline M 99-6,1 & 8,3 & 6,2 & 5,9 \\
\hline$M 99-3,8$ & 6,1 & 4,6 & 4,6 \\
\hline$M 99-4,7$ & 4,8 & 3,6 & 3,9 \\
\hline
\end{tabular}

Le tableau II présente les résultats des mesures des contraintes normales obtenus en 1995 (M 95) et 1999 (M99) ainsi que les résultats du premier modèle avec les vraies densités, les densités réduites et les résultats du second modèle. On rappelle que le second modèle diffère du premier non seulement en terme de densités mais aussi en terme de contraintes initiales.

Dans l'ensemble, on remarque un assez bon accord entre les mesures et les valeurs obtenues avec le premier modèle avec des densités réduites et avec le second modèle. Ce qui démontre que les transformations subies par un massif rocheux au cours de sa genèse ne sont probablement pas négligeables en terme de contraintes initiales et en terme de densités des divers faciès qui le constituent.

\section{2}

\section{Champ de contrainte}

Les modélisations bidimensionnelles réalisées ne permettent évidemment pas de nous renseigner sur l'orientation du repère principal des contraintes par rapport au Nord puisque par construction d'un modèle en déformations planes l'une des directions principales est orthogonale au plan du modèle.

Cependant, comme le montre la figure 10, qui représente l'évolution des contraintes principales le long du forage ID 180 déduite du modèle réduit, les deux composantes sub-horizontales sont pratiquement confondues. Dans ce cas, toutes les orientations par rapport au nord sont valables.

Par ailleurs, la comparaison avec les résultats de la campagne d'essais de 1995 (Fig. 4) montre que le modèle 1 réduit permet de retrouver assez correctement les contraintes principales $\sigma_{v}$ et $\sigma_{H}$. En revanche une grande divergence existe en ce qui concerne la contrainte sub-horizontale mineure qui est décroissante sur la figure 4 et croissante sur la figure 10.

Un autre accord encourageant concerne l'inclinaison par rapport à la verticale de la contrainte principale majeure $\sigma_{y}$ qui est de l'ordre de $10^{\circ}$ dans les deux campagnes de mesures et de l'ordre de $14^{\circ}$ dans les résultats du modèle (valeur pratiquement uniforme le long du forage ID 180 entre les profondeurs de 40 à $140 \mathrm{~m}$ ).

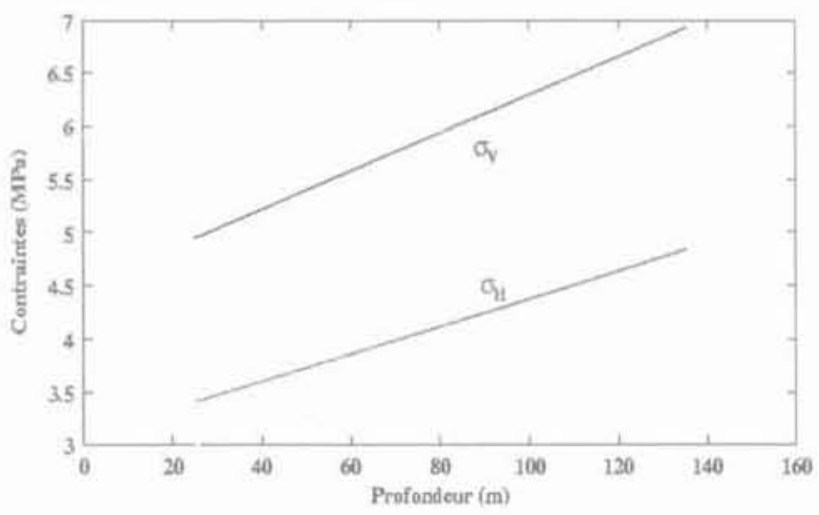

FG. 10 Contraintes principales en fonction de la profondeur (modèle 1 réduit). Principal stresses versus the depth (reduced model 1). 


\section{Conclusion}

A l'issue des deux campagnes d'essais menées sur le site de Tournemire, la dispersion et l'imprécision des résultats des mesures de contraintes semblent être une réalité du terrain. En effet, le fait de ne pas disposer dans les forages testés de fractures préexistantes d'orientations différentes, ne permet pas d'avoir une meilleure précision sur les amplitudes des contraintes même si on avait réalisé plus d'essais hydrauliques. Par ailleurs, l'existence de couches à différentes rigidités ainsi que la topographie marquée du massif ne facilitent pas l'interprétation des mesures.

Par ailleurs, les calculs des contraintes dans le massif de Tournemire soumis à la gravité ont permis de confirmer et de quantifier l'effet de la topographie sur le champ de contrainte. Ces calculs ont montré l'importance des poids volumiques des divers faciès sur les résultats obtenus. Il convient de noter que les valeurs des densités obtenues par essais de laboratoire et utilisées dans les calculs surestiment les contraintes. Ce qui démontre que la prise en compte de l'effet d'échelle sur ces paramètres est souvent indispensable pour avoir le meilleur accord entre les résultats théoriques et les mesures in situ. Cependant, ces calculs numériques n'ont pas permis de " trancher » entre les résultats différents des orientations des fractures testées et aussi entre les deux directions de la contrainte horizontale majeure données par les deux campagnes d'essais à cause du caractère bidimensionnel du modèle développé.

Par la présente étude on a cherché aussi à souligner que malgré les progrès de la géotechnique et malgré les efforts non négligeables des divers spécialistes des mesures in situ et des modélisations théoriques et numériques les informations qu'on peut obtenir sur le champ des contraintes dans un massif rocheux restent encore trop incertaines eu égard à la grande importance qu'ont de telles données lors de la modélisation de l'évolution de ces ouvrages et particulièrement lorsqu'il s'agit d'ouvrages pour le stockage de déchets nucléaires.

\section{REMERCIEMENTS}

Messieurs Antoinet. Bertrand et Ouvry d'ANTEA et messieurs Bert et Cornet de I'IPGP et Wileveau de GEOSTRESS ont réalisé les mesures in situ, qu'ils en soient vivement remerciés. Nous remercions éqalement messieurs Cabrera et Boisson qui ont suivi ces deux campagnes d'essais du côté IRSN.

\section{Bibliographie}

Amadei B., Stephansson O.--Rock stress and its measurements. Chapman \& Hall, 1997, p. 51-64.

Antoinet E.. Bertrand L. - Site de Tourne. mire (Aveyron): mesures des contraintes dans le massif par stimulation hydraulique de fractures en sondages. Rapport ANTEA n ${ }^{\circ}$ A05457. 1996.

Boisson J.-Y., Cabrera J.. De Windt L. Etude des écoulements dans un massif argileux, laboratoire souterrain de Tournemire. EUR 18338FR, 1988.

Burlet D. - Détermination du champ de contrainte régional à partir des tests hydrauliques en forages. These de IUniversité Paris VII, 1991.

Cabrera J., Beaucaire C., Bruno G., De windt L., Genty A., Ramambasoa N.. Rejeb A., Savoye S., Volant Ph. (2001)Projet Tournemire. Synthèse des programmes de recherche 1995-1999. Rapport IPSN/DPRE/SERGD 01-09, 2001.

Clarck J.B. - "A hydraulic process for increasing the $\mathrm{s}$ of wells $\mathrm{n}$. Trans. AIME, 186, 1949.

Cornet F.H., Valette B. - $\alpha$ In situ stress determination from hydraulic injection tests data ». J. Geophys, Res, vol. 89 B8, 1984, p. 11527-11537
Cornet F.H. Burlet D. - a Stress field determination in France by hydraulic tests in boreholes 3, J. Geophys. Res, vol. 97 . B8, 1992, p. 11829-11849.

Cornet F.H. - Détermination du champ de contrainte au voisinage du laboratoire souterrain de Tournemire. Rapport de ITPGP n' 98N33/0073, 2000.

Craig R.F. - Soil Mechanics. Van Nostrand Reinhold (UK) Co. Ltd, Wokingham. England, 1987, 4, ed.

Evans K.F., Engelder T., Plumb R.A. "Appalachian stress study. A detailed description of in situ variations in Devonian shales of the Appalachian plateau D. J. Geophys. Res., vol. 97. B6, 1989, p. 71329-7154.

Fairhurst C. - "Measurement of in sits rock stresses with particular reference to hydraulic fracturing w. Rock Mech. Enq. Geol. n० 11/3-4, 1964, p. 129-147.

Haimson B.C., Fairhurst C. $-\alpha$ In situ stress determination at great depth by means of hydraulic fracturing ". Proc. U.S. Symp. Rock Mech., 11, 1969, p. 559-584.

Hiramatsu Y.. Oka Y. - Stress around a shaft or level excavated in ground with a three-dimensional stress state. Mem. Fac of Eng. Kyoto Univ.. 24, 1962, p. 56-76.
Lekhnitskii S.G. - Theory of elasticity of an anisotropic elastic body. Holden Day. 1963.

Rejeb A - «Mechanical characterisation of the argillaceous Tournemire site (France) ». Proc. of the international conference on rock engineering techniques for site characterisation, Oxford and IBH Publishing Co. pvt. Ltd, 1999, p. 45-50.

Rejeb A., Humbert B., Vouille G. - Projet Tournemire. Programme mêcanique. Caractérisation du comportement mécanique au laboratoire. Rapport IPSN/SERGD 99/07, 1999

Sturgul J.R., Scheidegger A.E., Greenshpan Z. - «Finite element model of a mountain massif ». Geology, 4, 1976. p. $439-442$.

Wileveau Y. - Mesures du champ de contrainte dans les milieux complexes par la méthode HTPF. Naissance d'une sonde. Thèse de I'Université de Paris VII. 1997.

Wileveau Y - Mesures de contrainte in situ dans le tunnel de Tournemire (Aveyron. France). Rapport GEOSTRESS du $17 / 06 / 99$ au 25/06/99. 